\title{
An Intelligent Task Scheduling System for Electrical Appliances Using Particle Swarm Optimization
}

\author{
Justina Geoffrey Jaja ${ }^{1}$, Daniel Matthias², Nuka Nwiabu ${ }^{3}$ \\ Computer Science Department, Rivers State University, Port Harcourt, Nigeria ${ }^{1,2,3}$
}

\begin{abstract}
Over the previous years, electric power systems have experienced progressively visit stress condition because of consistently expanding power request, wasteful utilization of electric power age and transmission assets. Transmission line blackouts have been a typical reason for system stress conditions, which are conceivable to happen amid critical peak hours. Such occasions will cause a supply limit circumstance where falling disappointments and extensive territory power outages are conceivable. This research develops an intelligent task scheduling system for electrical appliances using particle swarm optimization. Object-oriented design methodology was used for system development. Particle Swarm Optimization (PSO) has been used to schedule domestic appliance to reduce consumption rate. PSO technique helps to balance load for each domestic appliance by scheduling load to the appliances. The load balance of domestic appliances such as freezer, water pump, water heater, tumble dryer and washing machine (energy consumption appliances), was modelled based on scheduled operation of several appliances at specific time according to customer lifestyle and priority of devices. System was implemented in Java programming language. The system was evaluated using weekly, monthly and yearly timeframe. The consumption rate of domestic appliances before and after optimization for weekly, monthly and yearly shows that optimization of the power consumed by domestic appliances reduced in all time frames, weekly with $12.8 \mathrm{KWH}$, monthly with $51.2 \mathrm{KWH}$ and yearly with $665.6 \mathrm{KWH}$.
\end{abstract}

Keywords: Energy consumption scheduling, inclining blockrates, price prediction, real-time pricing, wholesale electricitymarket, Task scheduling, Electrical appliances, Particle swam ptimization

\section{INTRODUCTION}

Intelligent System (IS) is a machine with an embedded, Internet-connected computer that has the capacity to gather and analyze data and communicate with other systems. In intelligent system, each of the systems use different types of technologies, components for managing electrical consumption rate like, Z-Wave, Zigbee and wired protocols.

These days, Utility organizations over the world have made different strides for effective utilization of power. The way toward watching, controlling and moderating power use in an organization or home is known as power management. It has been accounted that $40 \%$ of the worldwide power utilization happens inside home application [1]. However, Nigeria energy consumption has increasingly experienced an upward trend with over $23 \%$ increase in energy use since 2000 and Nigeria's energy consumption has consistently maintained an upward trend [2]. This takes into consideration direct correspondence between the service organization and the customer. Utility companies have higher electric charges amid peak periods. The work of power management systems in a local location lessens control bills for customers and peak requests. A Home Power Management (HPM) system in keen matrix empowers Demand Response (DR) and Demand Side Management (DSM) programs. Demand Response (DR) is characterized as "changes in power use by interest side assets from their typical utilization designs because of changes in the cost of power, or to motivating force installments intended to prompt lower power use are now and again of high discount advertise costs' or when system dependability is endangered" [3].

Task scheduling involves the assignment of an appropriate device to the appropriate task at the appropriate time while considering some constraints such as cost. Thus, the electricity produced in Nigeria varies between different hours of the day, between days of the week, and between seasons of the year. For this reason, it becomes a big challenge for the utility managing peak demands since electricity cannot be stored in bulk, so the utility has to match demand at any point in time. Demand response management has proven to be a significant way of reducing these peaks. Scheduling of electrical appliances and proper design of the electricity tariffs are some of the mechanisms used in demand response management to reduce these peaks. By these, the customer is motivated to participate in load balancing of the appliances with the aim of saving money on electric bills. [4] demonstrated a fully automated Energy Management System (EMS) using Reinforcement Learning (RL) techniques. The energy management and appliance scheduling problem is solved by Observe, Learn and Adapt (OLA) algorithm which adds more intelligence to EMS. The mechanism significantly reduces the cost and PAR, but User Comfort (UC) is compromised. 
International Journal of Advanced Research in Computer and Communication Engineering

Vol. 10, Issue 11, November 2021

DOI: $10.17148 /$ IJARCCE.2021.101105

This research proposes intelligent task scheduling system for electrical appliances using particle swarm optimization. An intelligent system will help to control and monitor the electronic appliances and reduce system load.

\section{TASK SCHEDULING}

Dynamic electricity costs are driving consumers to take advantage of more economical energy management by scheduling home appliances at times of lowest daily cost. However, most residential energy consumers have been accustomed to fixed-rate electricity and are not willing or able to conduct appliance scheduling, because of the time and mathematical calculations required. Time restrictions, knowledge of utility rate dynamics and math skills are impediments to average household users to correctly respond to dynamic energy costs [4]. Easy to use home appliance automation systems and energy decision support systems are highly desirable and they may be the keys to greater residential energy consumer use of more efficient energy scheduling.

Home appliances scheduling is a mathematical optimization problem, and thus shares many common elements such as objective(s), constraints, mathematical programing type, software or algorithm used to find solutions, global optimal solution or local optimal solution, with many other mathematical optimization problems.

Constraints are a series of equalities or inequalities which are necessary for restricting solutions to a feasible domain. For example, total electricity used by all appliances during a given period cannot exceed the allowed peak value, each appliance has its own electric specification, some appliances are closely related to each other in terms of the interval or time delay, and every user has his own appliance use preferences.

An optimization problem can formulate or deduced to a certain type of mathematical programming such as linear programming, nonlinear programming, deterministic programming, stochastic programming, integer programming (include mixed integer linear programming and binary integer linear programming) or continuous programming. In the case of a linear programming, all the constraints and the objective function are linear. Unlike deterministic programming, stochastic programming involves uncertainty in some parameters. Integer programming is characterized by some, or all of its variables are restricted to be integers, while continuous programming variables are continuous in specified ranges.

Mathematical programming can be solved using different algorithms and/or software. For integer linear programming, exact algorithms such as cutting plane methods, branch and bound, branch and cut, and branch and price are widely used [5]. Heuristic algorithms were developed by [6] to solve certain problems in optimization of household energy consumption. Heuristic algorithms prove more efficient in some large-scale problems, but each designed algorithm can only be effectively applied to limited types of problem and the solution may not be globally optimal. The programs AMPL, MATLAB, and SAS are effective programming tools to solve mathematical optimization problems, too. The CPLEX program provides Application Program Interfaces or Application Programming Interfaces (APIs) to C++, Java, and MATLAB. A free toolbox called YALMIP for modelling and optimization within the program MATLAB has become popular as a support for most mainstream optimizer and enable rapid algorithm development [7]. Use of a combination of MATLAB, YALMIP, and CPLEX MILP Solver can expedite the verification of the ideas associated with mathematical programming.

\section{RELATED WORK}

The term 'energy management' is defined as a function that ensures that energy generated at an instant in time within a system matches the energy consumed. However, as noted by [8] on aircraft the term load management is used to describe a function able to control just the loads, not the generators.

[9] presented research focusing on load management centres which are able to distribute energy to loads from multiple sources. The advantage of this system is to increase flexibility and reliability by allowing a bank of loads to be energized from multiple different sources, this however would come with an increased wiring requirement.

[10] describe advancements in load management technology. The initial form of load management was a list of loads that a pilot could shed in emergency situations by tripping a circuit breaker; this action could not be undone during flight. The same was true for the first automatic load shedding systems which would do what was previously done by a pilot.

[11] proposed a system which fully automated the shedding and re-connecting of loads by measuring the energy available from all sources then switching on loads up to the maximum energy available, prioritizing critical loads.

[12] proposed the use of particle swarm optimization technique (PSO) for prediction and monitoring against crude oil theft and security of oil and gas facility.

[13] prove that particle swarm optimization technique can be used to predict fire outbreak caused by electrical faults. This verifies that when the fault resistance is between $0.35 \mathrm{ohm}-0.0$-ohm fault resistance, there will be likelihood of danger occurring among all faults at the same time. 
The most advanced load management system was done by [14], which is focused on military ships but the algorithms would be similar to those on an aircraft. A system where loads can be shed and re-connected is proposed. This work improves on previous work by defining an algorithm that adapts the list of load priorities in real time rather than having a set list of loads to shed. This adaptive priority list is an improvement on previous work as it acknowledges that the priority of loads changes with time. Categories of load based on their modes of operation and their flexibility.

\section{FORCASTING DOMESTIC LOAD PROFILES FOR OPERATION}

Forecasting is making calculations or predictions of a near future event or condition based on analysis or study of historical data, events or observations. The relevance of accurate forecasting is evident in the meteorological system where significant progress has been achieved in computerized weather predictions. These forecasts are necessary for the aviation, industry, farmers, and governments. Similarly, load forecasts are very important in the electric industry, and are utilized by supply companies and network operators to anticipate the amount of energy needed to meet the demand and to predict transport flows on short and medium term. Forecasting assists companies in their decision-making processes, such as asset management, scheduling of generation units, and network congestion management. With increasing penetration of new technologies in the energy system, a good knowledge of the future electricity consumption and generation is crucial for the reliability of the network and operating strategies.

In the Netherlands, the "old" domestic energy meters are read once a year making it impossible for utilities to capture the dynamics of the daily, weekly and monthly domestic electricity consumptions. The introduction of smart metering systems provides the utilities with more data (15 minutes' averages of energy consumption). However real-time retrieval of customers' data is not allowed at the moment due to customer privacy concerns. By using historical meter data, load forecast models could be developed to estimate domestic demands. The estimation of domestic load can minimize the imbalance costs to the energy supplier, and provide more insight for the network operator on the state of his network. Additionally, domestic energy management systems can be optimized for peak-shaving, load shifting and other demand response applications with embedded forecasting models [16].

A. Load Forecasting Models

There are many load forecasting models depending on the time horizon and the complexity of the forecast. Forecasting models are broadly used for long, medium and short-term load forecasting. The accuracy of these models is a function of the forecasting techniques and the forecasted scenarios. Known forecasting techniques include: multiple regression, exponential smoothing, iterative re-weighted least squares, adaptive load forecasting, stochastic time series, fuzzy logic and artificial neural networks [17]. Important parameters often considered in such models are weather data, time factors, historical data, economic variables, and customer classes. A comparison of multiplicative load forecasting models reveals that the most accurate model for short and medium term forecasting has features according to equation (1):

$\mathrm{L}(\mathrm{t})=\mathrm{F}\{\mathrm{d}(\mathrm{t}), \mathrm{h}(\mathrm{t})\} . \mathrm{f}\{\mathrm{w}(\mathrm{t})+\mathrm{R}(\mathrm{t})\}$

Where,

$\mathrm{L}(\mathrm{t})$ is the forecasted load at time $\mathrm{t} ; \mathrm{d}(\mathrm{t})$ is the day of the week; $\mathrm{h}(\mathrm{t})$ is the hour of the day; $\mathrm{F}\{\mathrm{d}(\mathrm{t}), \mathrm{h}(\mathrm{t})\}$ is the daily and hourly component; $w(t)$ is the weather data; $f(w)$ is the weather factor; and $R(t)$ is a random error (noise), [15]

B. Particle Swarm Optimization

The original particle swarm optimization is a technique (PSO) such method updates each particle belonging to a population $\mathrm{S}$, defined as

$\mathrm{X} \_\mathrm{i}=\left[\mathrm{x} \_\mathrm{i} 1 \mathrm{x} \_\mathrm{i} 2 \ldots \mathrm{x} \_\mathrm{iD}\right]$ for dimension $\mathrm{D}$, by adjusting its velocity Vid in each dimension $\mathrm{d}$, for each new iteration $\mathrm{t}+1$ :

$\mathrm{v} \_\overline{i d}=\left[\bar{v} \_i d+\bar{c} \_1 * \llbracket\right.$ rand $\rrbracket \_1 *\left(p \_i d-x \_i d *\right)+c \_2 * \llbracket$ rand $\rrbracket \_2 *\left(\llbracket\right.$ pgbest $\left.\left.\rrbracket \_1 d-x \_i d\right)\right]$

Where,

$\mathrm{P}_{\mathrm{id}}$ represents the best local position recorded, for each dimension, and pgbest ${ }_{1 \mathrm{~d}}$ corresponds to the best global position obtained by the whole population, in dimension $d$. The two positive constants $c_{1}$ and $c_{2}$ correspond to the cognitive and social learning rates, respectively. The new particle position for the next generation in dimension $\mathrm{d}$, is thus obtained by adding the adjusted velocity to its current position:

x_id=x_id+v_(id)

\section{DOMESTIC APPLIANCES}

The load balance of domestic appliances such as freezer, dryer, water pump, water heater and washing machine (energy consumption appliances), can be modelled based on scheduled operation of several appliances at specific time according to customer lifestyle and priority of devices. The domestic appliance is shown in Figure 1. Domestic load model plays an important role in studying end user behaviours. 
Vol. 10, Issue 11, November 2021

DOI: $10.17148 /$ JJARCCE.2021.101105

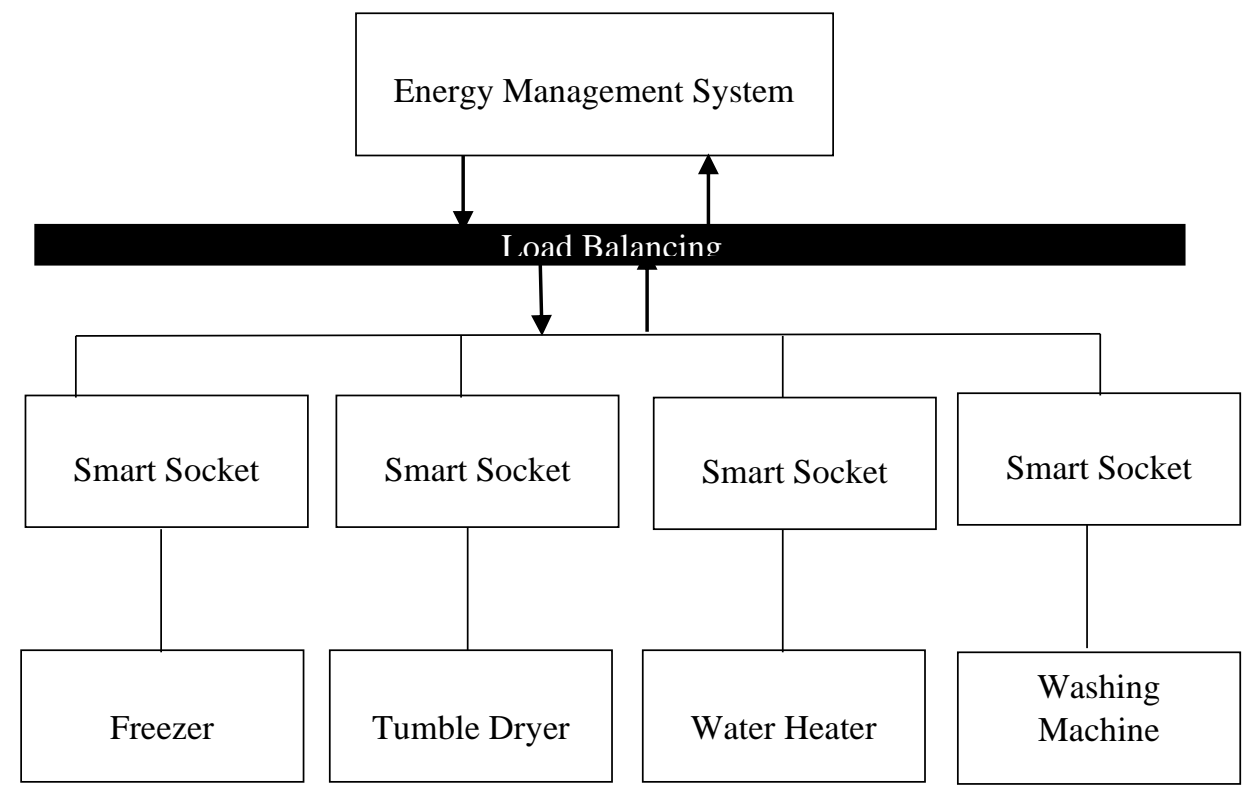

Fig 1. Domestic Appliances

Let $\mathrm{C}$ be the set of customers

Ac be the set of domestic appliances (e.g. Freezer, Tumble Dryer, washing machine and water heater, Water Pump) for each customer $\mathrm{c} \in \mathrm{C}$.

Each domestic appliance is scheduled to consume electricity or remain idle in each time interval (e.g., an hour) during the day. Residential customers may have different types of appliances namely, first, Type A (hard load), where certain appliances may have strict scheduling requirement, for example, a freezer should remain operational at all times, second, Type B (soft load),where many appliances may require constant amount of electricity consumption in a continuous fashion with flexible scheduling for a limited amount of time (e.g., washing machine) and lastly, Type C (soft load), where some appliances may need a fixed amount of electricity with irregular scheduling.

Let $\mathrm{X} \_\mathrm{n}^{\wedge} \mathrm{h}$ be the electricity consumption of a home appliance, $\mathrm{n}$ a member of domestic appliances in time interval $\mathrm{h}$.

Total electric energy $(\mathrm{kWh})$ consumed $(\lambda \mathrm{n})$ by appliance $\mathrm{n}$ during a day is given by:

$\lambda \_n=\sum(h=1)^{\wedge} H \llbracket\left(X \_n^{\wedge} h \rrbracket * 1 \_n^{\wedge} h^{*} m \_n \wedge h\right)$

In case of a hard load (Type A), for each hour $m \_n^{\wedge} h$ is equal to 1 if $1 \_n^{\wedge} h$ is equal to 1 .

In case of a soft load (Type C), if an appliance consumes $\lambda \mathrm{n}$ unit of energy (kWh) during a day then for each hour $\mathrm{m} \_\mathrm{n}^{\wedge} \mathrm{h}$ is $\{0,1\}$ if $1 \_\mathrm{n}^{\wedge} \mathrm{h}$ is equal to 1 .

When $\mathrm{m} \_\mathrm{n}^{\wedge} \mathrm{h}$ is equal to 1 , appliance $\mathrm{n}$ consumes $\mathrm{X} \_\mathrm{n}^{\wedge} \mathrm{h}$ units of electricity, otherwise it remains idle. Suppose that the House Energy has $\mathrm{N}$ home appliances, $\mathrm{N}=\{\mathrm{A} 1, \mathrm{~A} 2, \mathrm{~A} 3, \ldots \mathrm{AC}\}$, then the total electric energy consumed by appliance (Type C) per day must satisfy its target amount of electricity $1 \_n^{\wedge} \mathrm{h}$ can be calculated as follows;

$1 \_n^{\wedge} h=\sum(h=1)^{\wedge} H \llbracket\left(X \_n^{\wedge} h^{*} 1 \_n^{\wedge} h^{*} m \_n^{\wedge} h\right) \rrbracket$

Type $\mathrm{B}$ appliances consume electricity in a continuous fashion. Thus, in any time slot, if a type B equipment $n$ is scheduled to consume electricity, it will continue to consume electricity until the total consumption is equal to the target 1_n^ $\mathrm{T}$ such that;

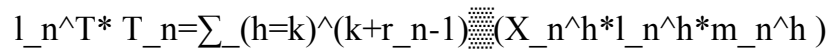

Where

$\mathrm{r} \_\mathrm{n}=\left(1 \_\mathrm{n}^{\wedge} \mathrm{T}\right) /\left(\mathrm{X} \_\mathrm{n}^{\wedge} \mathrm{h}\right)$ represents the number of time slots needed by appliance $\mathrm{n}$ to reach its target energy consumption $1 \_\mathrm{n}^{\wedge} \mathrm{T}$

Tn $(\mathrm{k})$ is a binary variable, which denotes the start time of type B appliance $\mathrm{n}$.

If Tn (k) equal to 1 , then appliance $\mathrm{n}$ starts consuming electricity in time slot $\mathrm{k}$. The load characteristics of domestic appliances are shown in Table1. 
Table I: LOAD CHARACTERISTIC

\begin{tabular}{llll}
\hline Load Type & Domestics Appliances & Energy (kw) & Daily Usage (hours) \\
\hline Un-Schedulable & Television & 0.15 & 15 \\
Appliances & Security cameras & 0.1 & 24 \\
& Computer & 0.2 & 12 \\
& Microwave oven & 0.5 & 6 \\
\multirow{3}{*}{ Schedulable Appliances } & Lights & 0.1 & 12 \\
& Tumble Dryer & 0.2 & 4 \\
& Washing Machine & 0.8 & 5 \\
& Water Pump & 0.4 & 2 \\
& Water Heater & 1 & 4 \\
& Freezer & 0.9 & 14 \\
\hline
\end{tabular}

The set of number of appliances for scheduling are denoted by $\mathrm{N}$ and their corresponding number of set of uninterruptible load profile for each appliance is denoted by ni for $\mathrm{i}=1,2, \mathrm{~N} . \mathrm{p} \_\mathrm{ij}{ }^{\wedge} \mathrm{k}$ represents load variable assigned to an appliance $\mathrm{i}$ having load phase $\mathrm{j}$ during time slot $\mathrm{k}$.

LetG_ij^kbinary decision variables to indicate whether a particular load profile is being processed and $\mathrm{H}_{-} \mathrm{ij} \mathrm{j}^{\wedge} \mathrm{k}$ binary decision variables to indicate whether a particular load profile is being idle. However, $G_{-} \mathrm{ij}^{\wedge} \mathrm{k}=1$ if an appliance $\mathrm{i}$ and its load phase $\mathrm{j}$ is being processed during the time slot $\mathrm{k}$, else $\mathrm{G}_{-} \mathrm{ij}{ }^{\wedge} \mathrm{k}=0$. Similarly, $\mathrm{H}_{-}(\mathrm{ij})^{\wedge} \mathrm{k}=1$ indicating an appliance $\mathrm{i}$, load phase $\mathrm{j}$ is already finished by time slot $\mathrm{k} . \mathrm{G}_{-} \mathrm{ij}{ }^{\wedge} \mathrm{k}$ and $\mathrm{H}_{-} \mathrm{ij}^{\wedge} \mathrm{k}$ are complementary and either binary variables G_ij^k=1 or $H_{-}(i j)^{\wedge} k=1$ such that;

$\mathrm{G}_{-} \mathrm{ij} \mathrm{j}^{\wedge} \mathrm{k}+\mathrm{H} \_\mathrm{ij}{ }^{\wedge} \mathrm{k}=1, \forall \mathrm{i}, \mathrm{j}, \mathrm{k}$

\section{METHODOLOGY}

Constructive research is a scientific procedure, that is based on the existing knowledge used in novel ways, with possibly adding a few missing links. The constructing proceeds through design thinking that makes projection into future envisaged solution (theory, artifact) and other knowledge gaps by building blocks to support the construction. Artifacts such as models, diagram, system designs, artificial languages and software development methods. The environmental factors are also taken into consideration in the design science approach. This model emphasizes the challenges and development needs of the environment. The task can be to create the desired states in the future of the company or companies. This is a key principle of a joint project, takes place within the interaction of the researchers and the company personnel. Research may play an important role in this. The task of research is to provide researched information in order to assist in the determination of the desired states of the companies and their descriptions.

\section{RESULTS}

Load scheduling varies at peak hours. The scheduled operation of five appliances at specific time is according to customer lifestyle and priority of devices. Freezer must remain operational at all times because of its usefulness in large and small home; each domestic appliance was scheduled to consume electricity or remain idle in each time interval during the day as shown in Table 4.1 .

TABLE II: WEEKLY OPERATIONAL STATE OF DOMESTIC APPLIANCES

\begin{tabular}{lccccc}
\hline $\begin{array}{l}\text { Domestic } \\
\text { Appliances }\end{array}$ & $\mathbf{0 1 : 5 1 : 0 1} \mathbf{A M}$ & $\mathbf{1 0 : 5 2 : 1 0 ~ P M}$ & $\begin{array}{c}\text { Consumption Time } \\
\mathbf{0 6 : 5 1 : 3 2} \mathbf{P M}\end{array}$ & $\mathbf{0 8 : 5 3 : 3 3 ~ A M}$ & $\mathbf{1 0 : 5 3 : 5 6 ~ A M ~}$ \\
\hline Freezer & On & On & On & On & On \\
water pump & Off & On & Off & Off & Off \\
water heater & Off & Off & On & Off & Off \\
washing machine & Off & Off & Off & On & Off \\
tumble dryer & Off & Off & Off & On & On \\
\hline
\end{tabular}


Vol. 10, Issue 11, November 2021

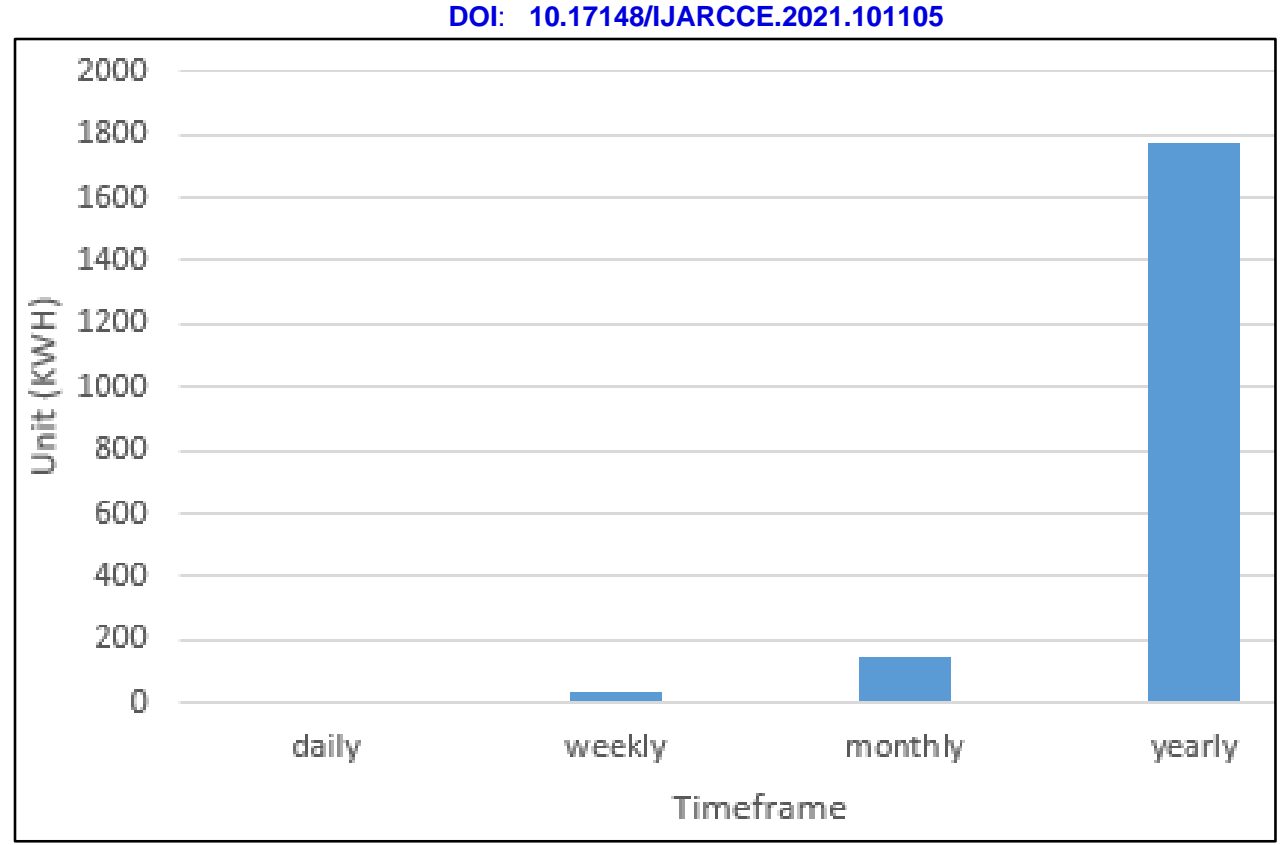

Figure 2:Power Units Consumed Before Optimization

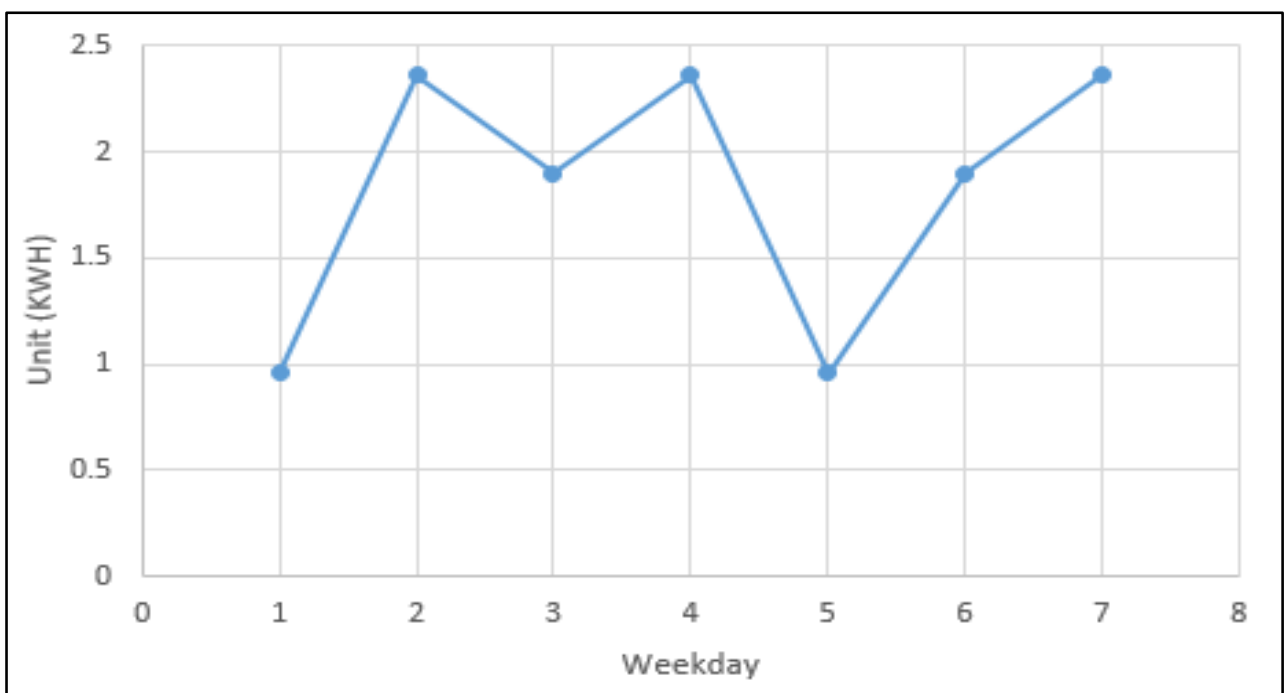

Figure 3:Power Units Consumed after Optimization

\section{CONCLUSION}

Due to the constantly expanding demand for energy, the wasteful use of electrical energy age and transmission assets, electrical energy systems have experienced stressful condition. Transmission line blackouts were a typical reason for system pressure conditions that may occur in critical peak hours. Such occasions will lead to a supply limit circumstance in which declining disappointments and extensive power outages on the territory are conceivable.

In this research, we have developed task scheduling system for electrical appliances using particle swarm optimization. Particle Swarm Optimization (PSO) has been used to schedule domestic appliance to reduce consumption rate. PSO technique helps to balance load for each domestic appliance by scheduling load to the appliances. The load balance of domestic appliances such as freezer, water pump, water heater, tumble dryer and washing machine (energy consumption appliances), was modelled based on scheduled operation of several appliances at specific time according to customer lifestyle and priority of devices.

Object-oriented methodology was adopted. It helped create a conceptual model of the energy management system.

The energy consumption appliances were based on scheduled operation of several appliances at specific time according to customer lifestyle and priority of devices. Each appliance has load percentage; the scheduling load to the appliances was done in peak hours. 


\section{International Journal of Advanced Research in Computer and Communication Engineering}

Vol. 10, Issue 11, November 2021

DOI: $10.17148 /$ IJARCCE.2021.101105

\section{REFERENCES}

[1]. M. N. Ullah, A. Mahmood, S.Razzaq, M. Ilahi, R.D. Khan, N. Javaid,A Survey of Different Residential Energy Consumption Controlling Techniques for Autonomous DSM in Future Smart Grid Communications. New York: arXiv preprint arXiv:1306.1134. 2013

[2]. A. O. Adeyemi, Electricity Consumption and Economic Growth in Nigeria. Ota: Journal of Business Management and Applied Economics, 2013.

[3]. A. Barbato, A. Capone; G. Carello; M. Delfanti; M. Merlo and A. Zaminga, House energy demand optimization in single and multiuser scenarios, Brussels: IEEE, 2011.

[4]. Z. Wen, D. O’Neill and H. Maei, Optimal demand response using device-based reinforcement learning. IEEE Trans. Smart Grid, 2015.

[5]. R. M. Lima and I. E. Grossmann, Computational advances in solving Mixed Integer Linear Programming problems. Pittsburgh: AIDAC,

2011.

[6]. L.D. Ha, S. Ploix, E.Zamai, and M.Jacomino, Tabu search for the optimization of household energy consumption. Waikoloa: IEEE 2006.

[7]. J. Löfberg, YALMIP: A toolbox for modeling and optimization in MATLAB. Taipei: IEEE. 2004

[8]. D. Schlabe, and J.Lienig, Energy management of aircraft electrical systems-state of the art and further directions, Bologna: IEEE, 2012.

[9]. D. A. Haak, and L. W. Messenger, Automatic electric load management centers. 9. 1990.

[10]. D. Schlabe, and J.Lienig, Energy management of aircraft electrical systems-state of the art and further directions, Bologna: IEEE, 2012.

[11]. D. T. Hambley, J. Jouper, S. Nellis, and M. A. Peabody, Load distribution and management system. US Patent, 6(46), 513. 2000.

[12] D. Matthias and K. E. Igbudu, Application of Particle Swarm Optimization Technique in Monitoring of Oil Theft and Oil Facility in The Nigeria Niger Delta, Nigerian Journal of Oil and Gas Technology 3(2), 2018

[13]. D. Matthias and O. E. Okpomo, Application of Particle Swarm Optimization Technique for Predicting Fire Outbreak Caused By Electrical Fault, European Journal of Computer Science and Information Technology, (7) 4, 5-15, 2019

[14]. Z. Ding; S. K. Srivastava; D. A. Carte and S. Suryanarayanan, Dynamic simulation-based analysis of a new load shedding scheme for a notional destroyer class shipboard power system. Arlington: IEEE, 2007.

[15]. H. Farhangi, The path of the smart grid. IEEE Power Energy Mag, 2010.

[16]. J. Hong, J. M. Cameron, J. M. Kim, and P. Tuohy, Demand-side management and control in buildings. Cambridge University Press, 2011.

[17]. H. Farhangi, The path of the smart grid. IEEE Power Energy Mag, 2010. 